\title{
Comparative Proteomic Analysis of Carbonylated Proteins from the Striatum and Cortex of Pesticide-Treated Mice
}

\author{
Christina Coughlan, ${ }^{1}$ Douglas I. Walker, ${ }^{2}$ Kelly M. Lohr, ${ }^{3}$ Jason R. Richardson, \\ Laura M. Saba, ${ }^{1}$ W. Michael Caudle, ${ }^{3}$ Kristofer S. Fritz, ${ }^{1}$ and James R. Roede ${ }^{1}$ \\ ${ }^{1}$ Department of Pharmaceutical Sciences, Skaggs School of Pharmacy and Pharmaceutical Sciences, University of Colorado, \\ Aurora, CO 80045, USA \\ ${ }^{2}$ Department of Medicine, Emory University, Atlanta, GA 30322, USA \\ ${ }^{3}$ Department of Environmental Health, Rollins School of Public Health, Emory University, Atlanta, GA 30322, USA \\ ${ }^{4}$ Department of Environmental and Occupational Medicine, Rutgers University Robert Wood Johnson Medical School, \\ Piscataway, NJ 08854, USA
}

Correspondence should be addressed to James R. Roede; james.roede@ucdenver.edu

Received 29 January 2015; Revised 27 May 2015; Accepted 30 May 2015

Academic Editor: Hélio Teive

Copyright ( $) 2015$ Christina Coughlan et al. This is an open access article distributed under the Creative Commons Attribution License, which permits unrestricted use, distribution, and reproduction in any medium, provided the original work is properly cited.

\begin{abstract}
Epidemiological studies indicate exposures to the herbicide paraquat (PQ) and fungicide maneb (MB) are associated with increased risk of Parkinson's disease (PD). Oxidative stress appears to be a premier mechanism that underlies damage to the nigrostriatal dopamine system in PD and pesticide exposure. Enhanced oxidative stress leads to lipid peroxidation and production of reactive aldehydes; therefore, we conducted proteomic analyses to identify carbonylated proteins in the striatum and cortex of pesticidetreated mice in order to elucidate possible mechanisms of toxicity. Male C57BL/6J mice were treated biweekly for 6 weeks with saline, PQ (10 mg/kg), MB (30 mg/kg), or the combination of PQ and MB (PQMB). Treatments resulted in significant behavioral alterations in all treated mice and depleted striatal dopamine in PQMB mice. Distinct differences in 4-hydroxynonenal-modified proteins were observed in the striatum and cortex. Proteomic analyses identified carbonylated proteins and peptides from the cortex and striatum, and pathway analyses revealed significant enrichment in a variety of KEGG pathways. Further analysis showed enrichment in proteins of the actin cytoskeleton in treated samples, but not in saline controls. These data indicate that treatmentrelated effects on cytoskeletal proteins could alter proper synaptic function, thereby resulting in impaired neuronal function and even neurodegeneration.
\end{abstract}

\section{Introduction}

Epidemiological studies of the past two decades have implicated environmental chemicals as possible causative factors for the development of Parkinson's disease (PD) [1-4]. Due to these observations, an environmental hypothesis of $\mathrm{PD}$ was derived stating that there are chemicals in the environment capable of selectively targeting the dopaminergic neurons of the substantia nigra $(\mathrm{SNpc})$ [5]. In a recent paper, Costello et al. determined that geographical areas with high use of the pesticides maneb $(\mathrm{MB})$ and paraquat $(\mathrm{PQ})$ had a high prevalence of PD [1]. In addition, PQ in combination with the dithiocarbamates, ziram and $\mathrm{MB}$, gives rise to an $80 \%$ increased risk of PD [6]. During the past decade, a number of studies have been conducted investigating the effects of $\mathrm{PQ}$ and $\mathrm{MB}$ on dopaminergic neurons in vivo. For example, the majority of these studies have shown that the combination of $\mathrm{PQ}$ and $\mathrm{MB}$ causes decreased striatal dopamine, decreased counts of tyrosine hydroxylase positive neurons in the SNpc, and impaired motor activity [7-12]. Increased lipid peroxidation has also been reported in this model [7, 13-15]. Additionally, in vitro studies have demonstrated that PQ and $\mathrm{MB}$ can alter cellular redox status $[16,17]$.

Oxidative stress is defined as an imbalance between oxidant production (free radicals and two-electron nonradicals, such as hydrogen peroxide) and antioxidant defenses 
(catalase, superoxide dismutase, peroxiredoxins, and glutathione peroxidase) [18]. There is strong evidence that points to the involvement of oxidative stress in the pathogenesis of PD. For example, etiologic factors like mitochondrial dysfunction, excitotoxicity, and inflammation all involve the production of reactive oxygen species (ROS). Additionally, postmortem analyses of PD brains have shown increased levels of iron, deficits in mitochondrial complex I activity, depleted glutathione (GSH), and increased lipid peroxidation [19-21].

Many reactive species are generated via the process of ROS-stimulated lipid peroxidation. This process can result in the accumulation of reactive aldehydes, such as 4-hydroxynonenal (4HNE). Reactive electrophiles covalently modify cellular macromolecules like proteins, DNA, and lipids. Of great importance to this study, lipid-derived electrophiles target nucleophilic amino acid residues, such as cysteine, histidine, and lysine [22-24]. This adduction leads to irreversible protein carbonylation, modulation of a protein's function (e.g., modification of the active site) [25-29], and/or aggregation and removal of the protein by cellular quality control mechanisms [28]. Additionally, free radicals can directly oxidize amino acid side chains and also lead to adduction by glycation products [30]. These events, as well as aldehyde adduction, all result in increased levels of protein carbonyls. Lastly, alterations in redox signaling and control during states of uncontrolled oxidant production and lipid peroxidation can ultimately lead to cellular toxicity and apoptosis [18].

Various investigators have published on the effects of PQ and MB in the mouse brain [9-11, 31]; however, the role of protein posttranslational oxidative modifications as an underlying factor for the initiation and progression of pesticide-induced PD remains unknown. Therefore, we performed a comparative proteomics study to investigate a brain region known to be affected by $\mathrm{PQ}$ and $\mathrm{MB}$, the striatum, as well as a lesser-studied region, the cortex. Upon examination, after 6 weeks of biweekly injections, striatal dopamine (DA), 3,4-dihydroxyphenylacetic acid (DOPAC), and homovanillic acid (HVA) were reduced and olfactory discrimination was altered. Protein extracts from the striatum and cortex showed distinct patterns of $4 \mathrm{HNE}$ modification. Cortex and striatal samples, when examined further for carbonylated proteins using LC-MS/MS-based proteomics, showed distinct toxicant-related effects. While PQ affected the striatum, as evidenced by a greater number of carbonylated proteins, $\mathrm{MB}$ treatment yielded the greatest number of carbonylated proteins in the cortex. Proteomic pathway analyses of these striatal and cortical adducted proteins showed an enrichment of many different KEGG pathways, particularly those involving the actin cytoskeleton. Together the results demonstrate that $\mathrm{PQ}$ and MB exposure affect olfactory discrimination, striatal catecholamine metabolism, and increased carbonylation of proteins involved in cytoskeletal dynamics.

\section{Materials and Methods}

2.1. Animal Treatments. All animal procedures were performed under a protocol approved by the Institutional
Animal Care and Use committee of Emory University. Male C57BL/6J mice (12 per group) were purchased from Jackson Laboratories and allowed to acclimate for one week prior to dosing. Animals were group housed in a group of 4 mice/cage and all treatment groups were present in each cage grouping. Mice were treated with saline $(0.9 \%)$, PQ $(10 \mathrm{mg} / \mathrm{kg}), \mathrm{MB}$ (30 mg/kg), or the combination of $\mathrm{PQ}$ and $\mathrm{MB}$ twice per week via i.p. injection, with a total injection volume not exceeding $0.25 \mathrm{~mL}$. These dose levels were chosen based upon previously published studies utilizing this model [711]. Animals receiving both $P Q$ and $M B$ received separate injections of $\mathrm{PQ}$ and $\mathrm{MB}$. All animals were weighed biweekly prior to dosing, and prior to sacrifice. Two weeks prior to the end of the study, all animals were separated and singly housed so animals could acclimate to their own cage for olfactory discrimination testing. At the completion of all behavioral testing, animals were euthanized via live decapitation. The striatum and cortex were dissected from the brains and snap frozen in liquid nitrogen until analysis. During the course of the study, animals in all groups gained weight and no significant differences in body weight were observed (data not shown). It must be noted, however, that two animals in the PQMB group died prior to the completion of dosing (week 3 and week 5), but no deaths were observed in the groups receiving $\mathrm{PQ}$ or $\mathrm{MB}$ alone.

2.2. Olfactory Discrimination. Olfactory discrimination was performed with modifications as previously described [32]. In summary, individual wooden blocks were placed in $50 \mathrm{~mL}$ conical tubes containing $1 \mathrm{~g}$ of soiled bedding from either the test animals' cages or strangers' cages. Blocks were placed in the tubes for $24 \mathrm{~h}$. For testing, the test mouse was presented with two blocks on opposing sides of the test cage: (1) a block scented with its own bedding and (2) a block scented with a stranger's bedding of the same sex. To determine the animal's ability to discriminate the scent of its home cage and a stranger's cage, the time spent in contact with each block was recorded for the $2 \mathrm{~min}$ trial. All trials were coded, videotaped, and timed by a blinded scorer. A total of 6-8 animals per treatment group were examined.

2.3. Locomotor Activity. All animals (10-12 per group) were tested for treatment-related impact on locomotor activity. Mice were placed in polycarbonate locomotor boxes $(25.4 \times$ $50.8 \times 25.4 \mathrm{~cm})$, and horizontal distance was quantified over time using Noldus Ethovision 3.0 (Noldus Information Technology, Wageningen, Netherlands) [33, 34]. General locomotion for treated and untreated mice was observed for a total of $4 \mathrm{~h}$. The first $30 \mathrm{~min}$ was considered the habituation period to ensure stabilization of the horizontal activity signal.

\subsection{High Performance Liquid Chromatography (HPLC) Deter-} mination of Striatal Neurochemistry. HPLC analyses of neurochemistry were performed using electrochemical detection as previously described $[33,35]$. Monoamine standards for DA, dihydroxyphenylacetic acid (DOPAC), and homovanillic acid (HVA) were purchased from Sigma. Mobile phase was 
obtained from ESA Inc. (Chelmsford, MA). Briefly, dissected striata from test animals (6-8 per group) were sonicated in $0.1 \mathrm{M}$ perchloric acid. Homogenates were centrifuged at $15,000 \times \mathrm{g}$ and the supernatant was filtered through a $0.22 \mu \mathrm{m}$ filter by centrifugation at $15,000 \times \mathrm{g}$. The supernatants were analyzed for levels of DA, DOPAC, and HVA. Quantification was made by reference to calibration curves made using individual standards.

2.5. SDS-PAGE and Western Blot. $50 \mu \mathrm{g}$ of each protein lysate (striatum and cortex) were separated via SDS-PAGE utilizing a $10 \%$ polyacrylamide gel. Transfer of the proteins onto PVDF membrane was performed using the BioRad Turbo Blotter with Trans-Blot Turbo 5x Transfer Buffer (BioRad). Blotting was performed on the mixed mw setting ( $8 \mathrm{~min})$. Blocking was performed for $20 \mathrm{~min}$ in Blotto $(0.5 \%$ nonfat dried milk/TBS-0.1\% Tween). Primary antibodies against 4HNE (generous gift from Dr. Dennis Petersen, University of Colorado) were diluted 1:1000 in Blotto and incubated with the blot overnight at $4^{\circ} \mathrm{C}$. After three washes for $10 \mathrm{~min}$ in $0.5 \% \mathrm{TBS} / 0.1 \%$ Tween, the blot was incubated with a horseradish peroxidase conjugated secondary antibody at 1:5000, diluted in Blotto. Clarity Western ECL Substrate (BioRad) was used to detect the HRP of the secondary antibody. Imaging was performed on the ChemiDoc MP imaging system and Image Lab software (BioRad). This experiment was conducted in three independent replications, and the image is a representative sample.

2.6. Biotin Hydrazide Derivatization of Carbonylated Protein. Hydrazide chemistry was employed to derivatize protein carbonyls from the samples (3-4 samples per treatment group) [36]. Mouse striatum and cortex lysates (400 $\mu \mathrm{g})$ were incubated in the dark with $5.0 \mathrm{mM}$ biotin hydrazide (Pierce, Rockford, IL) for 2 hours at $25^{\circ} \mathrm{C}$ with gentle rotation. Samples were then reduced with $5 \mathrm{mM}$ sodium borohydride for $60 \mathrm{~min}$ at $25^{\circ} \mathrm{C}$. Biotinylated proteins were filtered through Zeba Spin Desalting Columns (Pierce, Rockford, IL) to remove excess biotin hydrazide. Biotinylated samples were then applied to a streptavidin HP Spintrap column (GE Healthcare, Piscataway, NJ) for 60 minutes at $25^{\circ} \mathrm{C}$. Following 5 washes with $2.0 \mathrm{M}$ urea in PBS, biotinylated proteins were eluted in $0.1 \mathrm{M}$ ammonium hydroxide $(5 \mathrm{x})$, and samples were dried to completion using a vacuum centrifuge. Proteins were resuspended in Laemmli buffer and allowed to migrate approximately $1 \mathrm{~cm}$ into an SDS-PAGE gel and were Coomassie stained. The entire protein band was excised from the gel, digested with sequencing-grade trypsin (Promega, Madison, WI) in $50 \mathrm{mM}$ ammonium bicarbonate overnight at $37^{\circ} \mathrm{C}$ following a standard digestion protocol, and the resulting peptides were analyzed by LC-MS/MS.

Peptide separation was performed by nano-Advance Splitless nano-LC at a flow rate of $500 \mathrm{~nL} / \mathrm{min}$ with a gradient of 5 to $45 \%$ solvent B ( $90 \%$ acetonitrile, $0.1 \%$ formic acid) over $120 \mathrm{~min}$ on a $0.1 \mathrm{~mm} \times 150 \mathrm{~mm}$ Magic AQ C18 column (Michrome). The LC was coupled to an amaZon speed ETD ion trap mass spectrometer with captive spray ion source (Bruker Daltonics, Inc., Billerica, MA). The instrument was operated using data-dependent collision-induced dissociation (CID) MS/MS with a threshold for fragmentation at 100,000 counts (TIC). Data analysis was performed using Mascot (v 2.4, http://www.matrixscience.com) and Proteinscape (Bruker Daltonics). Peptide identifications were accepted if they could be established at greater than $95.0 \%$ probability, and protein identifications were accepted if they could be established at greater than $99.0 \%$ probability. All samples were run in duplicate. Searches resulted in the assignment of a UniProt (Universal Protein Resource, http://www.uniprot.org/) accession number that was used for subsequent bioinformatics analysis in ExPASy (http://www .expasy.org).

2.7. Functional Annotation and Enrichment. The carbonylated proteins identified in two mouse brain regions and the four treatment groups (8 protein lists) were functionally annotated using the Database for Annotation, Visualization, and Integrated Discovery (DAVID, version 6.7) [37, 38] based on UniProt IDs. KEGG pathways were examined for overrepresentation in carbonylated protein lists. KEGG pathways that were nominally significant $(P<0.01)$ in at least one of the 8 protein lists and not in all 8 protein lists were used in further analyses. An enrichment $P$ value was not calculated for an individual pathway if the protein list being analyzed did not contain at least two proteins from the pathway. When this occurred, a $P$ value of 1 was assumed. Hierarchical clustering was used to describe relationships among enriched pathways with respect to significance in the 8 protein lists; that is, pathways enriched in the same treatment groups and brain regions were clustered together. The distance measure for hierarchical clustering was calculated as one minus the Spearman correlation coefficient when $P$ values were converted to a binary indicator of significance $(P<0.01$ versus $P \geq 0.01)$. The hierarchical clustering analysis and the heatmap were generated using R Statistical Software (version 3.1.1).

\section{Results}

3.1. Locomotor Activity Was Not Affected by Pesticide Treatment. Previous studies employing this similar dosing regimen have reported deficits in motor function; therefore, we examined the animals for altered locomotor activity. The entire observation period was analyzed by hour, and no significant treatment-related decreases in locomotor activity were observed compared to saline controls. PQ treated animals did display hyperactivity in the first hour of testing; however, this observation was not statistically significant. In addition, the total number of ambulation instances did not differ significantly between treatment groups (Figure 1(a)). Nonmotor symptoms, such as olfactory problems, also occur in $\mathrm{PD}$; therefore, we next investigated pesticide-mediated effects on olfactory discrimination.

3.2. Pesticide Treatment Significantly Altered Olfactory Discrimination. A deficit in olfactory function is a hallmark clinical feature of PD. Interestingly, all pesticide treatments 


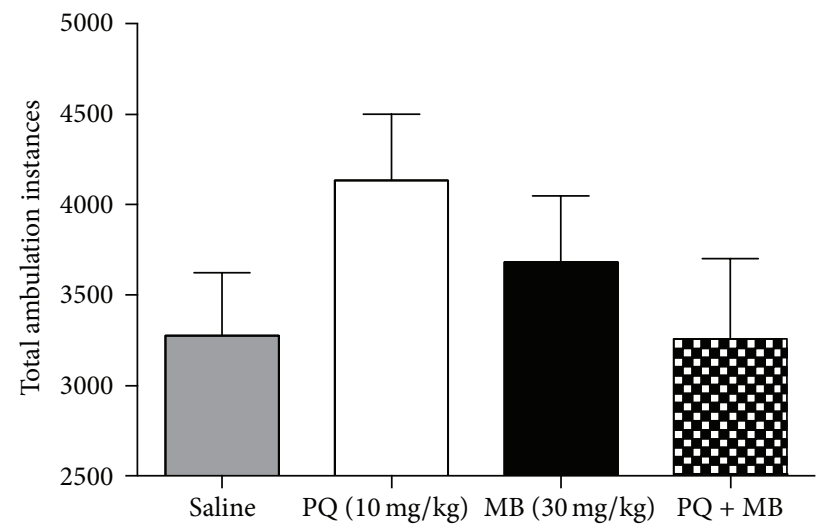

(a)

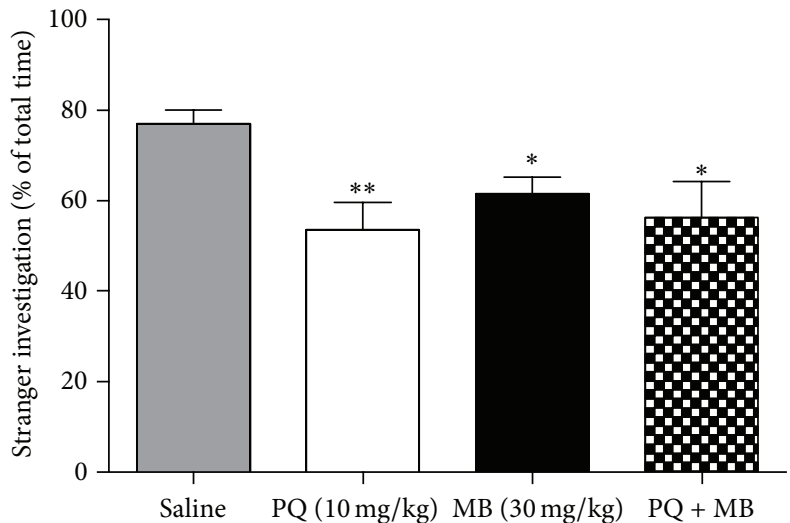

(b)

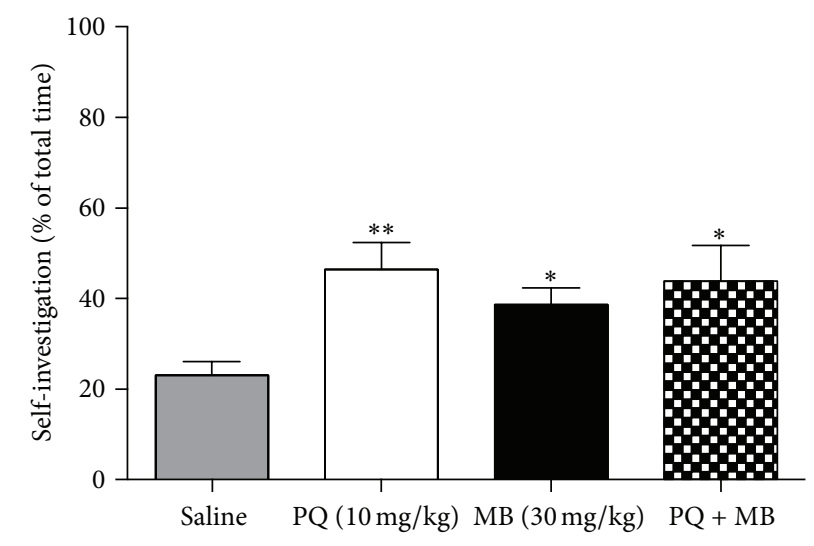

(c)

FIGURE 1: Pesticide treatment significantly alters olfactory discrimination, but not locomotor activity. PQ treated mice exhibited hyperactivity; however, these changes were not significant (a). Olfactory discrimination experiments revealed that all treatments caused a decrease in stranger investigation (b) and increased investigation of self (c). Data (mean \pm SEM) analyzed via one-way ANOVA and Dunnett's post hoc test $\left({ }^{* *} P<0.01 ;{ }^{*} P<0.05\right) . N=10-12$ animals per treatment group.

(PQ, MB, and PQMB) significantly affected olfactory discrimination as evidenced by reduced investigation of strangers (Figure 1(b)) and increased self-investigation time (Figure 1(c)). Previous work from our group has demonstrated the importance of striatal dopamine levels in mediating normal olfactory function $[32,39]$. Thus, we next investigated whether exposure to $\mathrm{PQ}$ and $\mathrm{MB}$ could affect dopamine content in the striatum.

\subsection{The Combination of $P Q$ and MB Significantly Decreased} Striatal DA and the Respective Metabolites (DOPAC and $H V A)$. Alterations in DA metabolism havebeen reported previously using this in vivo model $[9-11,40]$, and our behavioral studies show olfactory deficits; therefore, we investigated the striatal levels of DA and its proximal metabolites, DOPAC and HVA. The combination treatment (PQMB) reduced DA levels and its metabolites (DOPAC and HVA) in the striatum (Figure 2). PQ also reduced DOPAC. MB alone did not alter the striatal concentration of any of the compounds tested.

3.4. Extracts of Striatum and Cortex Displayed Distinct Patterns and Intensity of 4 HNE Protein Adducts. The alterations to striatal DA observed in our model could be the result of more subtle damage to the function of the dopamine terminals in the striatum that are not easily detected by immunoblot and stereology. The generation of ROS is a hallmark of PD pathology and has been shown to affect specific aspects of neuronal function [41, 42]; therefore, we aimed to assess mechanisms that may alter nerve function, such as pesticide-mediated oxidative protein adducts. To investigate this, extracts from the striatum (known to be affected by PQMB) and cortex (not known to be altered) ( $n=3$ /treatment) were immunoblotted for 4 HNE-modified proteins (Figure 3(a)). The staining pattern was distinct for each brain region and, compared to the striatum, the cortex displayed a much more intense staining for all the $4 \mathrm{HNE}$ bands observed. This translates into an increased quantity of $4 \mathrm{HNE}$-adducted proteins basally present in the cortex compared to the striatum. Additionally, this blot shows that the striatum exhibits treatment-related increases in adducts compared to the cortex (Figure $3(\mathrm{~b})$ ). $4 \mathrm{HNE}$ protein adducts represent one of many possible protein carbonylation products; therefore, proteomics investigations were performed on the cortex and striatum of these mice to identify carbonylated proteins and to elucidate the metabolic pathways affected. 


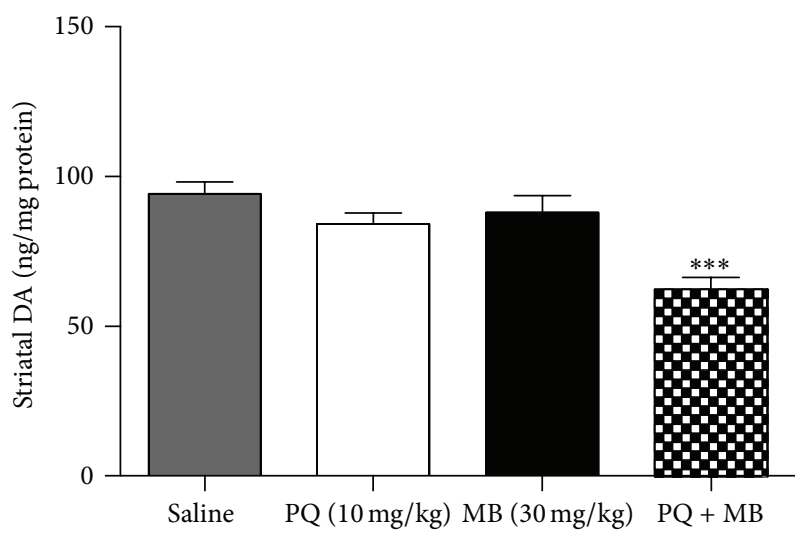

(a)

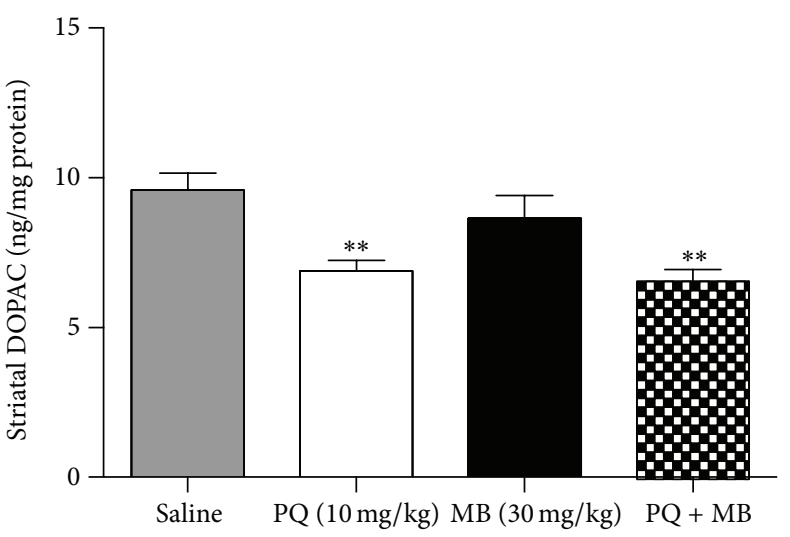

(b)

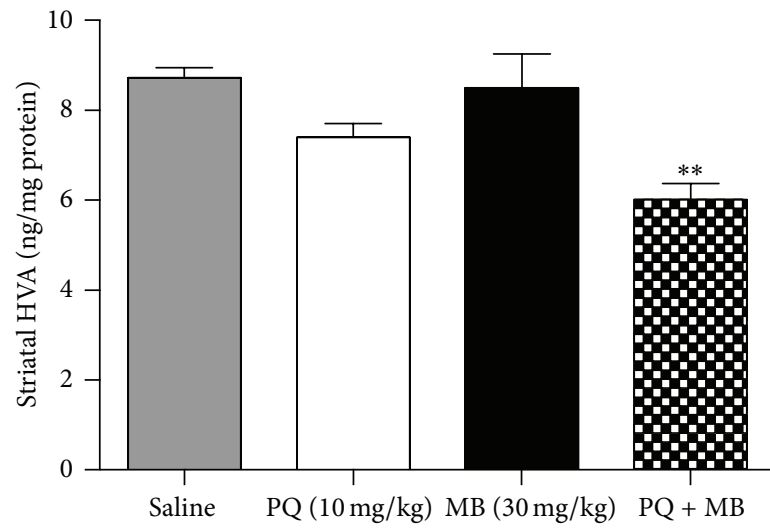

(c)

FIGURE 2: The combination of PQ and MB significantly decreases striatal DA, as well as its downstream metabolites. DA (a), DOPAC (b), and HVA (c) were quantified in striatal samples using HPLC and electrochemical detection. Data analyzed via one-way ANOVA and Dunnett's post hoc test $\left({ }^{* *} P<0.01 ;{ }^{*} P<0.05\right) . N=6-8$ animals per treatment group.

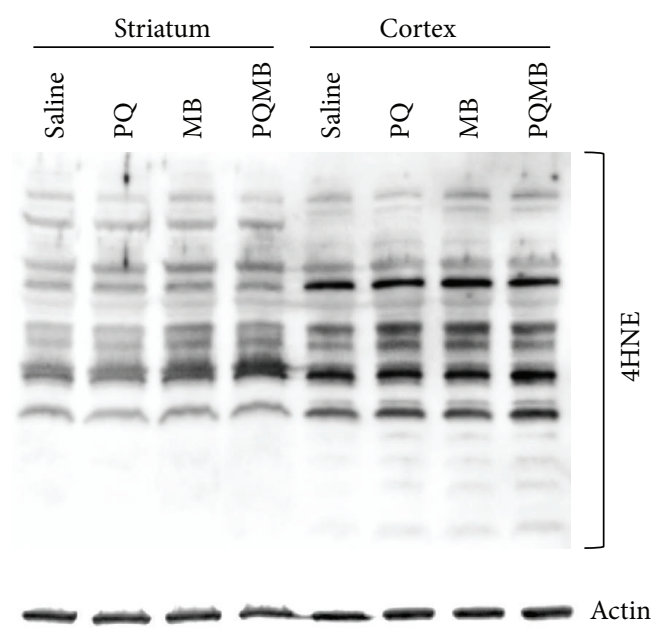

(a)

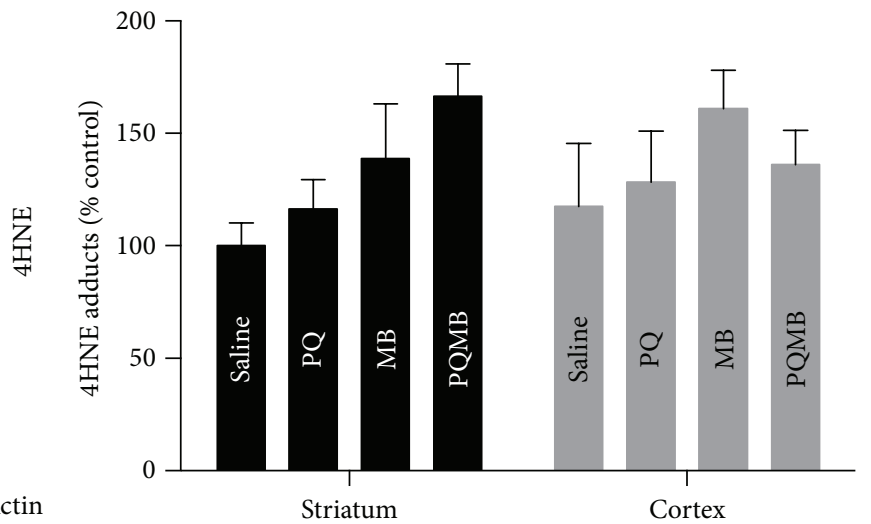

(b)

FIGURE 3: Extracts of striatum and cortex display distinct patterns and intensity of $4 \mathrm{HNE}$ protein adducts. Extracts $(N=3)$ were immunoblotted using an antibody specific for 4HNE-modified protein (a). Quantification of 4HNE-modified proteins shows treatmentrelated changes in striatal HNE adducts (b). Western blots were conducted in triplicate. 

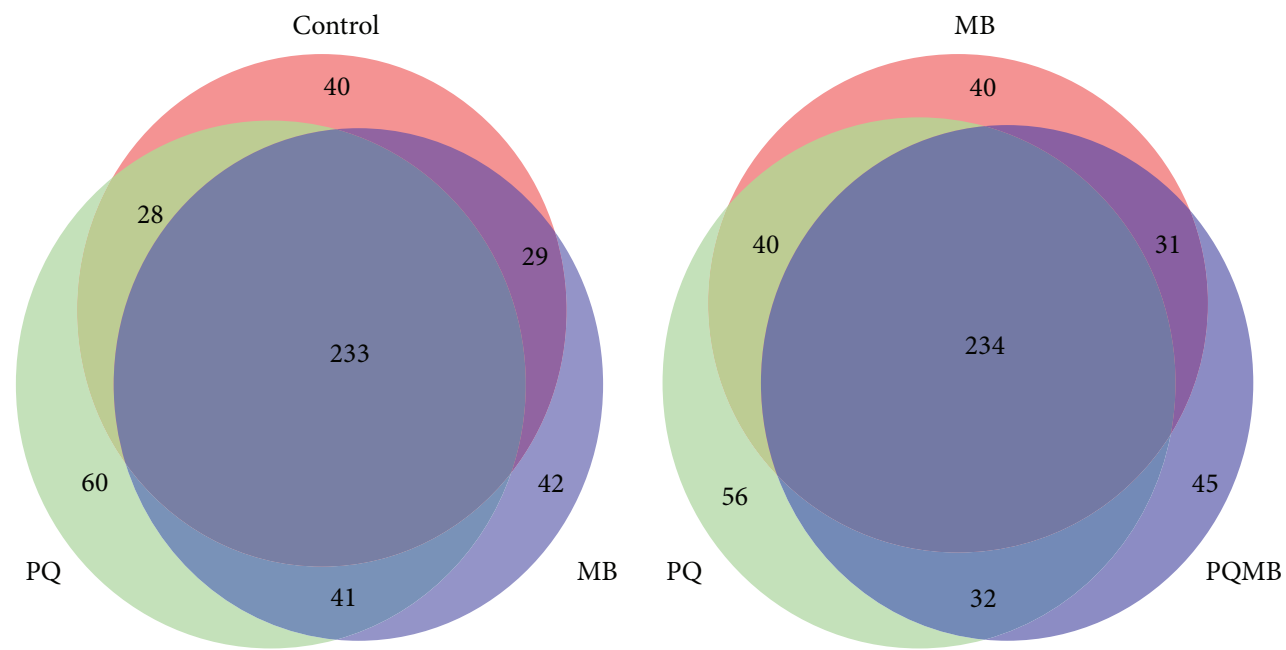

(a)
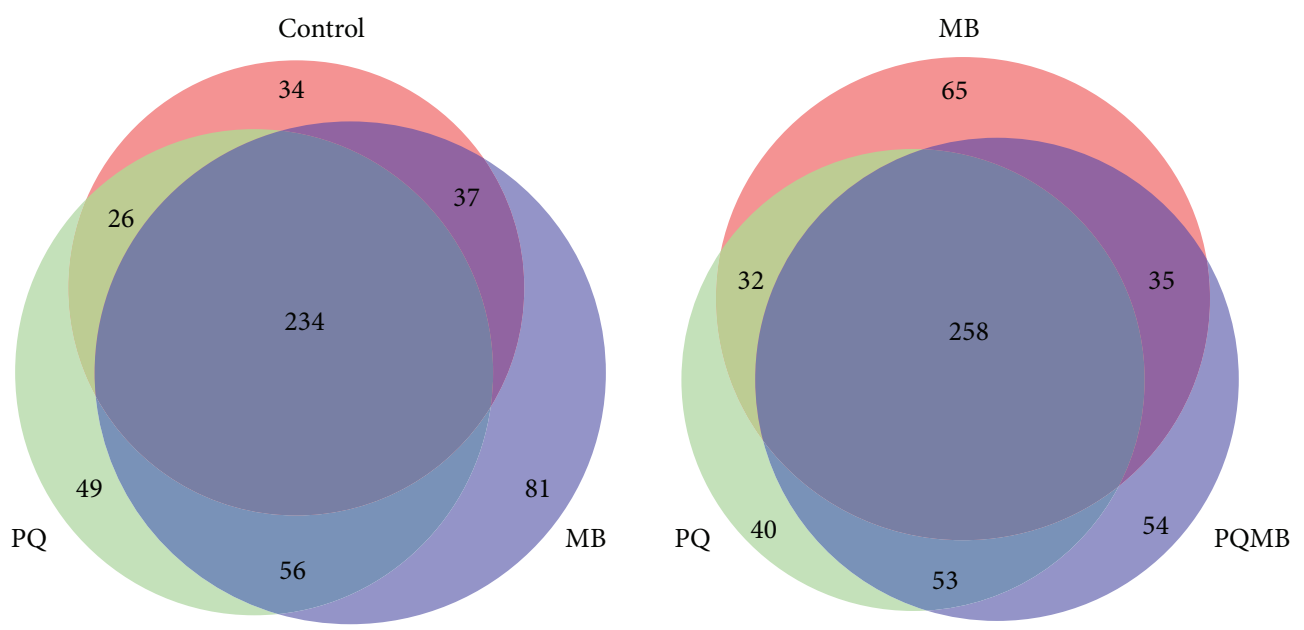

(b)

FIGURE 4: Proteins detected in each brain region display toxicant specific effects. Venn diagrams display the number of proteins that were identified to be unique or shared by the treatment groups in the striatum (a) and the cortex (b).

3.5. Proteomic Investigation of Carbonylated Proteins Showed Toxicant Specificities for Brain Regions. To enrich our samples for carbonylated proteins, a pull-down assay utilizing biotin hydrazide conjugation and streptavidin purification was utilized. Eluates from the pull-down were then digested with trypsin and analyzed via LC-MS/MS. Results of the proteomics analyses of the striatum revealed that a greater number of carbonylated proteins were detected in the PQ treated samples (364) compared to saline (332), MB (346), and PQMB (342) (Figure 4(a)). With respect to a comparison of saline versus $\mathrm{PQ}, \mathrm{MB}$, or $\mathrm{PQMB}$ treatment, $\mathrm{PQ}$ treated animals had the largest number of proteins unique to the treatment. Analyses of cortex samples were slightly different when compared to the striatum. A greater number of carbonylated proteins were detected in MB-treated samples (408) compared to saline (332), PQ (367), and PQMB (400) (Figure 4(b)). Similar to PQ in the striatum, MB-treated animals had the largest number of unique proteins (81) compared to the other conditions. These results demonstrate that PQ has greater effects on the striatum, while MB alters the cortex to a larger degree.

3.6. Bioinformatics Analyses Showed That Protein Carbonylation Affects Cellular Junctions, Cytoskeleton, and the Proteasome. To gain further insight into the molecular processes altered due to carbonylation, protein lists from each treatment group were analyzed using a freely available bioinformatics program, the Database for Annotation, Visualization, and Integrated Discovery (DAVID) [37, 38]. The majority of the carbonylated proteins in each of the 8 experimental conditions (average $=96.6 \%$ ) were found in the DAVID database. Thirty KEGG pathways were significantly $(P<$ 0.01 ) overrepresented in at least one of the eight protein lists. Of the 30, 8 KEGG pathways were overrepresented in all 8 protein lists and were excluded from further analyses. The other 22 KEGG pathways were clustered based on their $P$ values across the 8 protein lists (Figure 5). Hierarchical clustering analysis revealed two major clusters of KEGG 

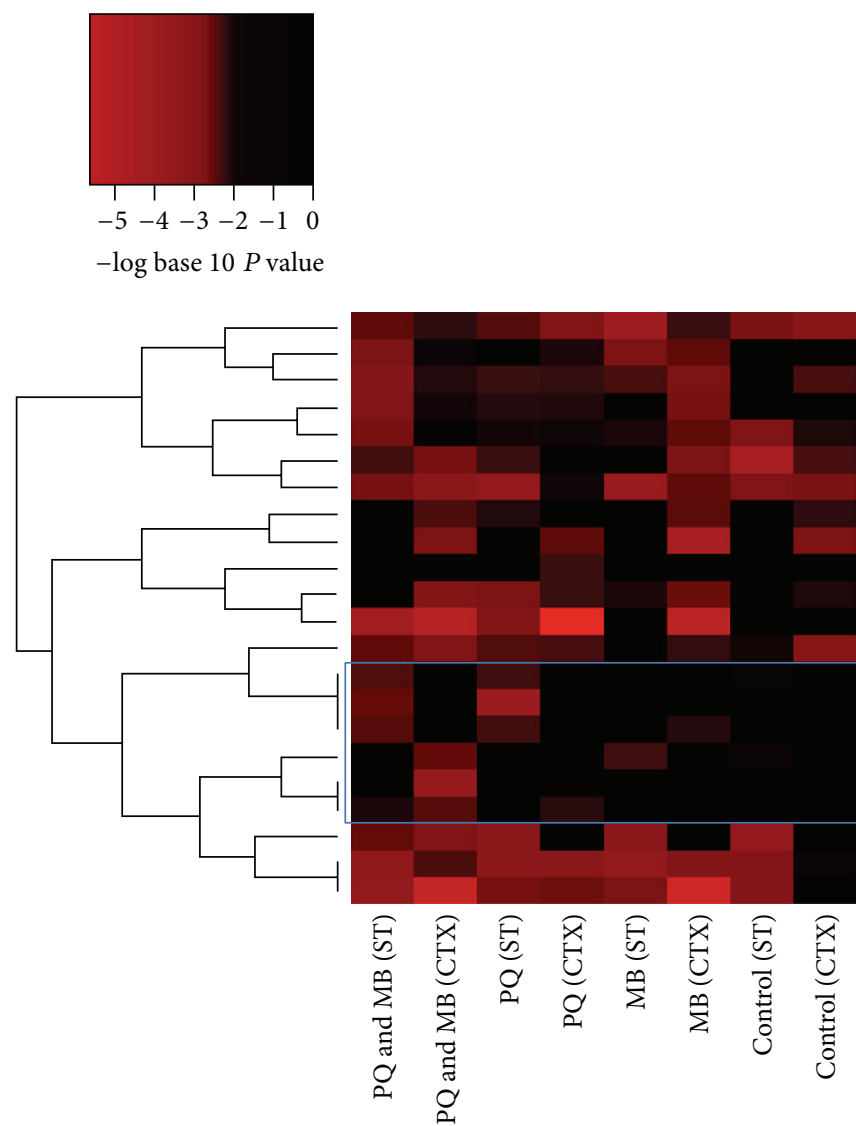

Cardiac muscle contraction

Aldosterone-regulated sodium reabsorption

Fructose and mannose metabolism

Galactose metabolism

Alanine, aspartate, and glutamate metabolism

Butanoate metabolism

Propanoate metabolism

Amyotrophic lateral sclerosis (ALS)

Ribosome

Starch and sucrose metabolism

Fc gamma R-mediated phagocytosis

Pentose phosphate pathway

Neurotrophin signaling pathway

Tight junction

Adherens junction

Calcium signaling pathway

Arginine and proline metabolism

Proteasome

Regulation of actin cytoskeleton

Valine, leucine, and isoleucine degradation

Gap junction

Long-term potentiation

FIGURE 5: KEGG pathways enriched for carbonylated proteins by pesticide treatment and brain region. The carbonylated proteins identified in two mouse brain regions, striatum (ST) and cortex (CTX), were functionally annotated using DAVID Tools. KEGG pathways were examined for overrepresentation in carbonylated protein lists obtained for each of the four treatment groups, maneb (MB), paraquat (PQ), paraquat and maneb combined (PQMB), and controls, and in each brain region separately. KEGG pathways that were nominally significant $(P<0.01)$ in at least one of the 8 protein lists and not in all 8 protein lists are included in the graphic. The colors of the heatmap range from white $(P>0.01)$ to bright red based on the negative $\log$ base 10 transformation of the $P$ value. A $P$ value of 1 was used when the KEGG pathway was represented by less than two proteins in the list. KEGG pathways (rows) are ordered based on hierarchical clustering where the distance measure was calculated as one minus the Spearman correlation coefficient when $P$ values were converted to a binary indicator of significance $(P<0.01$ versus $P \geq 0.01)$.

pathways. One cluster (top) contains 7 pathways, while the second cluster (bottom) contains 15 pathways. This top cluster of 7 pathways consists of pathways detected in almost all of the treatments and controls. The second cluster contains pathways that were not enriched in the controls. Specifically, the boxed area shows 6 pathways significantly enriched in the PQMB group in both striatum and cortex, with little or no enrichment in the saline treated mice. Pathways with poor enrichment in the saline controls included tight junctions, adherens junctions, calcium signaling, Arg and Pro metabolism, the proteasome, and regulation of the actin cytoskeleton.

\section{Discussion}

The purpose of this study was to investigate a possible role for protein carbonylation in a PQ and MB model of neurotoxicity. After 6 weeks of biweekly treatments, we observed reductions in striatal levels of DA and its metabolites, alterations in olfactory discrimination, increases in $4 \mathrm{HNE}$ protein adducts in the cortex and striatum, and carbonylation of proteins enriched in cellular junctions, calcium signaling, and cytoskeletal pathways.

Olfactory deficits were observed in all of the pesticidetreated groups, which is the first time this phenotype has been characterized in this model. In addition to the striatum's role in olfaction, activation of the amygdala and hippocampus is intricately involved in forming olfactory memories [43, 44]. Given that these brain regions are often targeted in neurodegenerative diseases, these olfactory deficits are novel and intriguing, especially in light of that fact that altered olfactory discrimination is observed in animals with reduced monoamine storage capacity [39]. Because the striatum is involved in olfaction, our observation of increased $4 \mathrm{HNE}$ protein adducts and decreased dopamine levels in the striatum points to a potential role for protein carbonylation as a 
modifier of olfactory pathways. While decreased striatal DA was only observed in the PQMB group, all treated animals displayed olfactory deficits, implying the involvement of additional mechanisms. Finally, it should be noted that these treatments might be effective in damaging the olfactory bulb, and this mechanistic possibility will be evaluated in future studies.

A potential mechanism for these olfactory deficits may involve the cortex. Our data demonstrate distinct patterns of 4 HNE-modified proteins in this brain region. Impaired olfactory discrimination has been reported in response to cortical injury and atrophy $[45,46]$. Interestingly, the loss of the sense of smell is one of the earliest signs of PD, a symptom reported years before the onset of motor symptoms [47]. Additionally, olfactory deficits are being investigated as a diagnostic approach to detect and discriminate between depression and Alzheimer's disease (AD) in the elderly [44], given that olfactory deficits are also an early symptom of $\mathrm{AD}$ [48]. Together, these findings corroborate with PQ and $\mathrm{MB}$ treatments affecting neuronal pathways and point to the importance of carbonylation in the modification of neuronal activity and function.

These studies also demonstrate treatment-mediated changes in striatal DA and DA metabolite levels. This observation appears to be a pathological hallmark of this pesticide model. For example, Thiruchelvam and colleagues reported a decrease in striatal DA of approximately $20 \%$ in the first description of this model [11]. Additional studies from the Cory-Slechta laboratory and others have described decreases in striatal DA of $20-35 \%[8,10,12]$. In our study, we observed that the combination treatment (PQMB) decreased the DA level, along with the corresponding metabolites DOPAC and HVA, approximately 35\%. These observations support the PQMB treatment regimen as a model system for striatal DA depletion and PD.

The striatum and cortex were shown to have distinct patterns of $4 \mathrm{HNE}$ adducts in response to $\mathrm{PQ}, \mathrm{MB}$, and PQMB treatment. This is a novel and important observation in light of the fact that lipid peroxidation is increased in neurodegenerative diseases like PD, AD, and Down syndrome [49]. Increased protein carbonylation due to PQMB has been observed previously [7]; however, this study investigated changes in whole brain instead of the specific regions described here, and these investigators did not employ proteomics to evaluate possible proteins and metabolic pathways affected. In further support of our findings, increased $4 \mathrm{HNE}$ protein adducts have been detected in a C. elegans model of a common genetic form of PD involving the gene LRRK2 [50]. This study also supports the involvement of ROS and aldehyde adducts in the development of PD. As redefined by Jones [18], oxidative stress involves a disruption of redox signaling and control. Cellular redox signaling and control employs reactive Cys residues in proteins to act as "sulfur switches" that control a host of processes like macromolecular interactions, allosteric control, and active site "onoff" switches [51]. Previous studies have demonstrated the importance of Cys modification by $4 \mathrm{HNE}$ in regard to altering protein function $[25-27,52,53]$. Additionally, proper function of DAT is reliant on redox sensitive Cys residues, as evidenced by studies involving oxidants that result in Cys oxidation and conformational changes [54, 55]. VMAT2 function is also affected by modification of redox sensitive Cys residues [56]. TH activity is also inhibited by redox thiols. For example, TH activity was found to be inhibited by toxicant- or oxidative stress-mediated glutathionylation of six of seven Cys residues [57], which may account for decreases in striatal DA observed in our study. These data indicate that oxidative stress induced by pesticide exposure leads to enhanced lipid peroxidation and aldehyde production. Thus, these aldehydes and/or ROS are capable of altering redox control of enzymes and transporters responsible for proper functioning of dopaminergic neurons.

Further investigation of the proteomic data for significant KEGG pathway enrichment revealed two distinct clusters of pathways. The smallest cluster contained 7 KEGG pathways and the majority of the pathways were significantly enriched in the saline controls as well as the toxicant treated animals. This data indicates that these particular pathways are possible targets of reactive aldehydes produced during normal metabolic processes. The second cluster of pathways is more variable and contains a block of 6 pathways that have little to no significant enrichment in the saline treated controls. These pathways include tight junctions, adherens junctions, calcium signaling, Arg and Pro metabolism, the proteasome, and regulation of the cytoskeleton. Three of these six pathways, tight junctions, adherens junctions, and regulation of actin cytoskeleton, involve cytoskeletal proteins (actin) and various regulatory proteins. The actin cytoskeleton is involved in many neuronal processes such as axon growth, arborization, and synaptic function [58]. Posttranslational modifications of the cytoskeleton, such as phosphorylation and glycation, have been shown to cause alteration to axonal structure and function. These changes may lead to neuronal degeneration, inappropriate targeting of neurons, or migration issues $[59,60]$. Additionally, actin is involved in maintaining the proper structure and function of dopaminergic neurons [61]. In our study, we detected carbonylation of actin isoforms ( $\beta$-actin and $\gamma$-actin), as well as members of the Arp2/3 complex (ARC1A, ARPC2, and ARPC4) and RHOA and WASF1, which are involved in F-actin nucleation and axon arborization. The impaired olfactory discrimination that we observed may be a consequence of alterations in the actin cytoskeleton; however, future studies are being conducted to specifically evaluate the toxicant-mediated effects on the actin cytoskeleton and axonal/synapse morphology.

Actin and its regulatory proteins are also involved in cellular junctions, adherens and tight. Tight junctions function as "gates" that prevent paracellular passage of ions and solutes between cells [62]. The main structures responsible for the barrier properties of the blood-brain barrier (BBB) are tight junctions [63]; therefore, any unfavorable alteration in proteins involved in this function may render this barrier permeable to solutes that do not normally passively diffuse across. PQ remains a controversial toxicant in regard to its ability to traverse the BBB $[64,65]$. Altered BBB function could provide an explanation for the enhanced neurotoxicity 
observed by previous investigators when mice are treated with PQ and MB in combination [10-12].

\section{Conclusion}

This study further demonstrates that PQ and MB treatments negatively affect the brain and central nervous system. This was evidenced by treatment-related deficits in olfactory discrimination and decreased striatal dopamine. Additionally, we showed that there are distinct patterns of $4 \mathrm{HNE}$ modification in the cortex compared to the striatum. Further analyses of these modified proteins revealed treatmentrelated enrichment in various KEGG pathways. In particular, a cluster of 6 pathways (most of which involved the actin cytoskeleton) was enriched in treated samples, but not saline controls. These data indicate possible dysfunction in actin cytoskeletal dynamics, which would lead to altered axonal growth, arborization, and synaptic function. Future experiments are required to confirm the mechanistic involvement of actin cytoskeletal dynamics in the observed pesticidemediated neurotoxicity.

\section{Conflict of Interests}

The authors declare that there is no conflict of interests regarding the publication of this paper.

\section{Acknowledgments}

The authors would like to thank Dr. James Galligan for his critical review of the paper. The work presented here was supported by grants from the National Institutes of Health, NIH/NIEHS R00 ES022266 (J. R. Roede), R01 ES021800 (J. R. Richardson), P30 ES019776 (W. M. Caudle), and NIH/NIAAA R01 AA022146 (K. S. Fritz).

\section{References}

[1] S. Costello, M. Cockburn, J. Bronstein, X. Zhang, and B. Ritz, "Parkinson's disease and residential exposure to maneb and paraquat from agricultural applications in the central valley of California," American Journal of Epidemiology, vol. 169, no. 8, pp. 919-926, 2009.

[2] B. R. Ritz, A. D. Manthripragada, S. Costello et al., "Dopamine transporter genetic variants and pesticides in Parkinson's disease," Environmental Health Perspectives, vol. 117, no. 6, pp. 964969, 2009.

[3] A. Priyadarshi, S. A. Khuder, E. A. Schaub, and S. S. Priyadarshi, "Environmental risk factors and Parkinson's disease: a metaanalysis," Environmental Research, vol. 86, no. 2, pp. 122-127, 2001.

[4] D. B. Hancock, E. R. Martin, G. M. Mayhew et al., "Pesticide exposure and risk of Parkinson's disease: a family-based casecontrol study," BMC Neurology, vol. 8, article 6, 2008.

[5] D. A. Drechsel and M. Patel, "Role of reactive oxygen species in the neurotoxicity of environmental agents implicated in Parkinson's disease," Free Radical Biology and Medicine, vol. 44, no. 11, pp. 1873-1886, 2008.
[6] A. Wang, S. Costello, M. Cockburn, X. Zhang, J. Bronstein, and B. Ritz, "Parkinson's disease risk from ambient exposure to pesticides," European Journal of Epidemiology, vol. 26, no. 7, pp. 547-555, 2011.

[7] A. Kachroo and M. A. Schwarzschild, "Allopurinol reduces levels of urate and dopamine but not dopaminergic neurons in a dual pesticide model of Parkinson's disease," Brain Research, vol. 1563, pp. 103-109, 2014.

[8] N. K. Singhal, G. Srivastava, D. K. Patel, S. K. Jain, and M. P. Singh, "Melatonin or silymarin reduces maneb- and paraquatinduced Parkinson's disease phenotype in the mouse," Journal of Pineal Research, vol. 50, no. 2, pp. 97-109, 2011.

[9] M. Thiruchelvam, B. J. Brockel, E. K. Richfield, R. B. Baggs, and D. A. Cory-Slechta, "Potentiated and preferential effects of combined paraquat and maneb on nigrostriatal dopamine systems: environmental risk factors for Parkinson's disease?" Brain Research, vol. 873, no. 2, pp. 225-234, 2000.

[10] M. Thiruchelvam, A. McCormack, E. K. Richfield et al., "Agerelated irreversible progressive nigrostriatal dopaminergic neurotoxicity in the paraquat and maneb model of the Parkinson's disease phenotype," European Journal of Neuroscience, vol. 18, no. 3, pp. 589-600, 2003.

[11] M. Thiruchelvam, E. K. Richfield, R. B. Baggs, A. W. Tank, and D. A. Cory-Slechta, "The nigrostriatal dopaminergic system as a preferential target of repeated exposures to combined paraquat and maneb: implications for Parkinson's disease," Journal of Neuroscience, vol. 20, no. 24, pp. 9207-9214, 2000.

[12] X. Li, K. Matsumoto, Y. Murakami, Y. Tezuka, Y. Wu, and S. Kadota, "Neuroprotective effects of Polygonum multiflorum on nigrostriatal dopaminergic degeneration induced by paraquat and maneb in mice," Pharmacology Biochemistry and Behavior, vol. 82, no. 2, pp. 345-352, 2005.

[13] J. Prakash, S. K. Yadav, S. Chouhan, and S. P. Singh, "Neuroprotective role of withania somnifera root extract in manebparaquat induced mouse model of parkinsonism," Neurochemical Research, vol. 38, no. 5, pp. 972-980, 2013.

[14] S. P. Gupta, S. Patel, S. Yadav, A. K. Singh, S. Singh, and M. P. Singh, "Involvement of nitric oxide in maneb- and paraquatinduced Parkinson's disease phenotype in mouse: is there any link with lipid peroxidation?" Neurochemical Research, vol. 35, no. 8, pp. 1206-1213, 2010.

[15] S. Patel, V. Singh, A. Kumar, Y. K. Gupta, and M. P. Singh, "Status of antioxidant defense system and expression of toxicant responsive genes in striatum of maneb- and paraquat-induced Parkinson's disease phenotype in mouse: mechanism of neurodegeneration," Brain Research, vol. 1081, no. 1, pp. 9-18, 2006.

[16] J. R. Roede, J. M. Hansen, Y.-M. Go, and D. P. Jones, "Maneb and paraquat-mediated neurotoxicity: involvement of peroxiredoxin/thioredoxin system," Toxicological Sciences, vol. 121, no. 2, pp. 368-375, 2011.

[17] J. R. Roede and D. P. Jones, "Thiol-reactivity of the fungicide maneb," Redox Biology, vol. 2, no. 1, pp. 651-655, 2014.

[18] D. P. Jones, "Redefining oxidative stress," Antioxidants and Redox Signaling, vol. 8, no. 9-10, pp. 1865-1879, 2006.

[19] P. Jenner and C. W. Olanow, "Understanding cell death in Parkinson's disease," Annals of Neurology, vol. 44, no. 3, supplement 1, pp. S72-S84, 1998.

[20] T. M. Dawson and V. L. Dawson, "Molecular pathways of neurodegeneration in Parkinson's disease," Science, vol. 302, no. 5646, pp. 819-822, 2003. 
[21] A. Yoritaka, N. Hattori, K. Uchida, M. Tanaka, E. R. Stadtman, and Y. Mizuno, "Immunohistochemical detection of 4hydroxynonenal protein adducts in Parkinson disease," Proceedings of the National Academy of Sciences of the United States of America, vol. 93, no. 7, pp. 2696-2701, 1996.

[22] H. Esterbauer, R. J. Schaur, and H. Zollner, "Chemistry and biochemistry of 4-hydroxynonenal, malonaldehyde and related aldehydes," Free Radical Biology and Medicine, vol. 11, no. 1, pp. 81-128, 1991.

[23] J. A. Doorn and D. R. Petersen, "Covalent modification of amino acid nucleophiles by the lipid peroxidation products 4hydroxy-2-nonenal and 4-oxo-2-nonenal," Chemical Research in Toxicology, vol. 15, no. 11, pp. 1445-1450, 2002.

[24] J. A. Doorn and D. R. Petersen, "Covalent adduction of nucleophilic amino acids by 4-hydroxynonenal and 4-oxononenal," Chemico-Biological Interactions, vol. 143-144, pp. 93-100, 2003.

[25] J. R. Roede, D. L. Carbone, J. A. Doorn, O. V. Kirichenko, P. Reigan, and D. R. Petersen, "In vitro and in silico characterization of peroxiredoxin 6 modified by 4-hydroxynonenal and 4-oxononenal," Chemical Research in Toxicology, vol. 21, no. 12, pp. 2289-2299, 2008.

[26] K. S. Fritz, J. J. Galligan, R. L. Smathers et al., "4-hydroxynonenal inhibits SIRT3 via thiol-specific modification," Chemical Research in Toxicology, vol. 24, no. 5, pp. 651-662, 2011.

[27] D. S. Backos, K. S. Fritz, J. R. Roede, D. R. Petersen, and C. C. Franklin, "Posttranslational modification and regulation of glutamate-cysteine ligase by the $\alpha, \beta$-unsaturated aldehyde 4hydroxy-2-nonenal," Free Radical Biology and Medicine, vol. 50, no. 1, pp. 14-26, 2011.

[28] J. J. Galligan, K. S. Fritz, D. S. Backos et al., "Oxidative stressmediated aldehyde adduction of GRP78 in a mouse model of alcoholic liver disease: functional independence of ATPase activity and chaperone function," Free Radical Biology and Medicine, vol. 73, pp. 411-420, 2014.

[29] R. L. Smathers, K. S. Fritz, J. J. Galligan et al., "Characterization of 4-HNE modified L-FABP reveals alterations in structural and functional dynamics," PLoS ONE, vol. 7, no. 6, Article ID e38459, 2012.

[30] D. A. Butterfield, M. Perluigi, and R. Sultana, "Oxidative stress in Alzheimer's disease brain: new insights from redox proteomics," European Journal of Pharmacology, vol. 545, no. 1, pp. 39-50, 2006.

[31] P. Desplats, P. Patel, K. Kosberg et al., "Combined exposure to Maneb and Paraquat alters transcriptional regulation of neurogenesis-related genes in mice models of Parkinson's disease," Molecular Neurodegeneration, vol. 7, no. 1, article 49, 2012.

[32] J. L. Tillerson, W. M. Caudle, J. M. Parent, C. Gong, T. Schallert, and G. W. Miller, "Olfactory discrimination deficits in mice lacking the dopamine transporter or the D2 dopamine receptor," Behavioural Brain Research, vol. 172, no. 1, pp. 97-105, 2006.

[33] W. M. Caudle, J. R. Richardson, M. Z. Wang et al., "Reduced vesicular storage of dopamine causes progressive nigrostriatal neurodegeneration," Journal of Neuroscience, vol. 27, no. 30, pp. 8138-8148, 2007.

[34] J. M. Bradner, T. A. Suragh, W. W. Wilson et al., "Exposure to the polybrominated diphenyl ether mixture DE-71 damages the nigrostriatal dopamine system: role of dopamine handling in neurotoxicity," Experimental Neurology, vol. 241, no. 1, pp. 138147, 2013.

[35] M. Sheleg, C. L. Yochum, G. C. Wagner, R. Zhou, and J. R. Richardson, "Ephrin-A5 deficiency alters sensorimotor and monoaminergic development," Behavioural Brain Research, vol. 236, no. 1, pp. 139-147, 2013.

[36] J. J. Galligan, R. L. Smathers, K. S. Fritz, L. E. Epperson, L. E. Hunter, and D. R. Petersen, "Protein carbonylation in a murine model for early alcoholic liver disease," Chemical Research in Toxicology, vol. 25, no. 5, pp. 1012-1021, 2012.

[37] D. W. Huang, B. T. Sherman, and R. A. Lempicki, "Bioinformatics enrichment tools: paths toward the comprehensive functional analysis of large gene lists," Nucleic Acids Research, vol. 37, no. 1, pp. 1-13, 2009.

[38] D. W. Huang, B. T. Sherman, and R. A. Lempicki, "Systematic and integrative analysis of large gene lists using DAVID bioinformatics resources," Nature Protocols, vol. 4, no. 1, pp. 44-57, 2009.

[39] T. N. Taylor, W. M. Caudle, K. R. Shepherd et al., "Nonmotor symptoms of Parkinson's disease revealed in an animal model with reduced monoamine storage capacity," Journal of Neuroscience, vol. 29, no. 25, pp. 8103-8113, 2009.

[40] M. Thiruchelvam, E. K. Richfield, B. M. Goodman, R. B. Baggs, and D. A. Cory-Slechta, "Developmental exposure to the pesticides paraquat and maneb and the Parkinson's disease phenotype," NeuroToxicology, vol. 23, no. 4-5, pp. 621-633, 2002.

[41] A. H. V. Schapira, "Mitochondria in the aetiology and pathogenesis of Parkinson's disease," The Lancet Neurology, vol. 7, no. 1, pp. 97-109, 2008.

[42] Y. Zhang, V. L. Dawson, and T. M. Dawson, "Oxidative stress and genetics in the pathogenesis of parkinson's disease," Neurobiology of Disease, vol. 7, no. 4, pp. 240-250, 2000.

[43] D. A. Deems, R. L. Doty, R. G. Settle et al., "Smell and taste disorders, a study of 750 patients from the University of Pennsylvania Smell and Taste Center," Archives of Otolaryngology: Head and Neck Surgery, vol. 117, no. 5, pp. 519-528, 1991.

[44] N. Marine and A. Boriana, "Olfactory markers of depression and Alzheimer's disease," Neuroscience and Biobehavioral Reviews, vol. 45C, pp. 262-270, 2014.

[45] E.-Y. Lee, P. J. Eslinger, G. Du, L. Kong, M. M. Lewis, and $\mathrm{X}$. Huang, "Olfactory-related cortical atrophy is associated with olfactory dysfunction in Parkinson's disease," Movement Disorders, vol. 29, no. 9, pp. 1205-1208, 2014.

[46] K. L. Radomski, Q. Zhou, K. J. Yi, and M. L. Doughty, "Cortical contusion injury disrupts olfactory bulb neurogenesis in adult mice," BMC Neuroscience, vol. 14, article 142, 2013.

[47] A. Haehner, T. Hummel, and H. Reichmann, "Olfactory loss in Parkinson's disease," Parkinson's Disease, vol. 2011, Article ID 450939, 6 pages, 2011.

[48] G. Coronas-Sámano, W. Portillo, V. B. Campos, G. MedinaAguirre, R. Paredes, and S. Diaz-Cintra, "Deficits in odorguided behaviors in the transgenic $3 \mathrm{xTg}-\mathrm{AD}$ female mouse model of Alzheimer's disease," Brain Research, vol. 1572, pp. 1825, 2014.

[49] M. Shichiri, "The role of lipid peroxidation in neurological disorders," Journal of Clinical Biochemistry and Nutrition, vol. 54, no. 3, pp. 151-160, 2014.

[50] F. Di Domenico, R. Sultana, A. Ferree et al., "Redox proteomics analyses of the influence of co-expression of wild-type or mutated LRRK2 and Tau on C. elegans protein expression and oxidative modification: relevance to parkinson disease," Antioxidants \& Redox Signaling, vol. 17, no. 11, pp. 1490-1506, 2012.

[51] D. P. Jones, "Radical-free biology of oxidative stress," The American Journal of Physiology-Cell Physiology, vol. 295, no. 4, pp. C849-C868, 2008. 
[52] B. J. Stewart, J. R. Roede, J. A. Doorn, and D. R. Petersen, "Lipid aldehyde-mediated cross-linking of apolipoprotein B100 inhibits secretion from HepG2 cells," Biochimica et Biophysica Acta, vol. 1791, no. 8, pp. 772-780, 2009.

[53] C. Wakita, T. Maeshima, A. Yamazaki et al., "Stereochemical configuration of 4-hydroxy-2-nonenal-cysteine adducts and their stereoselective formation in a redox-regulated protein," The Journal of Biological Chemistry, vol. 284, no. 42, pp. 2881028822, 2009.

[54] S. U. Park, J. V. Ferrer, J. A. Javitch, and D. M. Kuhn, "Peroxynitrite inactivates the human dopamine transporter by modification of cysteine 342: potential mechanism of neurotoxicity in dopamine neurons," Journal of Neuroscience, vol. 22, no. 11, pp. 4399-4405, 2002.

[55] N. H. Chen and M. E. A. Reith, "Structure and function of the dopamine transporter," European Journal of Pharmacology, vol. 405, no. 1-3, pp. 329-339, 2000.

[56] D. S. Thiriot and A. E. Ruoho, "Mutagenesis and derivatization of human vesicle monoamine transporter 2 (VMAT2) cysteines identifies transporter domains involved in tetrabenazine binding and substrate transport," The Journal of Biological Chemistry, vol. 276, no. 29, pp. 27304-27315, 2001.

[57] C. R. Borges, T. Geddes, J. T. Watson, and D. M. Kuhn, "Dopamine biosynthesis is regulated by S-glutathionylation. Potential mechanism of tyrosine hydroxylast inhibition during oxidative stress," Journal of Biological Chemistry, vol. 277, no. 50, pp. 48295-48302, 2002.

[58] J. C. Nelson, A. K. H. Stavoe, and D. A. Colón-Ramos, "The actin cytoskeleton in presynaptic assembly," Cell Adhesion and Migration, vol. 7, no. 4, pp. 379-387, 2013.

[59] Y. Kise and D. Schmucker, "Role of self-avoidance in neuronal wiring," Current Opinion in Neurobiology, vol. 23, no. 6, pp. 983989, 2013.

[60] M. J. Renzi, T. L. Wexler, and J. A. Raper, "Olfactory sensory axons expressing a dominant-negative semaphorin receptor enter the CNS early and overshoot their target," Neuron, vol. 28, no. 2, pp. 437-447, 2000.

[61] J. K. Juranek, "Axonal transport of cytoskeleton and cytoskeleton regulatory cargo proteins in neurodegenerative disorders," OA Anatomy, vol. 1, no. 3, p. 22, 2013.

[62] A. Hartsock and W. J. Nelson, "Adherens and tight junctions: structure, function and connections to the actin cytoskeleton," Biochimica et Biophysica Acta-Biomembranes, vol. 1778, no. 3, pp. 660-669, 2008.

[63] H. Wolburg and A. Lippoldt, "Tight junctions of the bloodbrain barrier: development, composition and regulation," Vascular Pharmacology, vol. 38, no. 6, pp. 323-337, 2002.

[64] G. W. Miller, "Paraquat: the red herring of Parkinson's disease research," Toxicological Sciences, vol. 100, no. 1, pp. 1-2, 2007.

[65] R. M. LoPachin and T. Gavin, "Response to 'Paraquat: the red herring of Parkinson's disease research"' Toxicological Sciences, vol. 103, no. 1, pp. 219-223, 2008. 


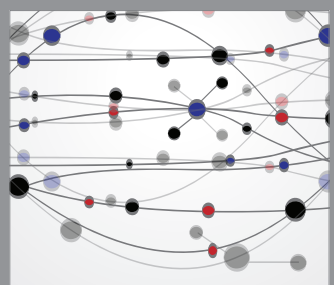

The Scientific World Journal
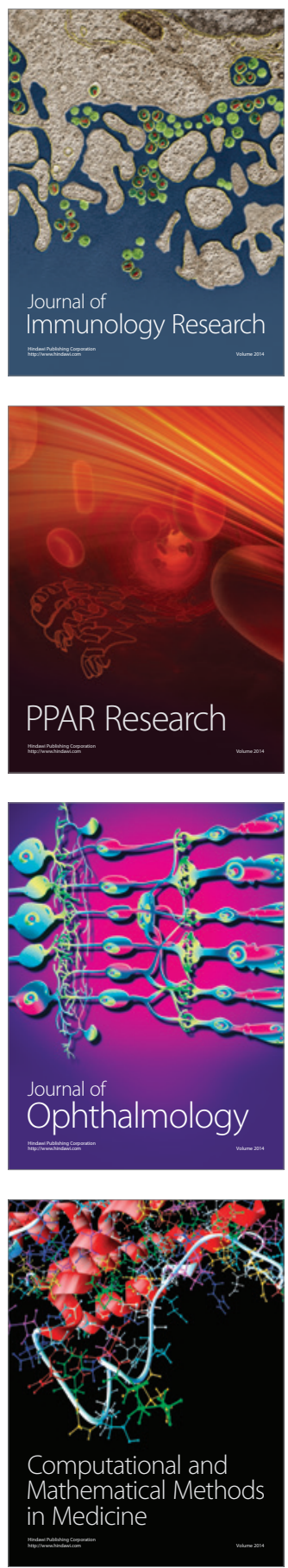

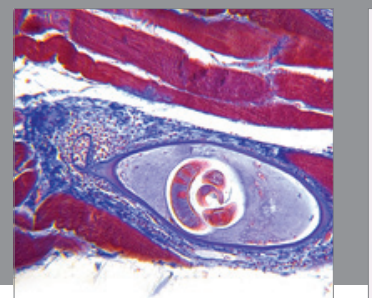

Gastroenterology

Research and Practice
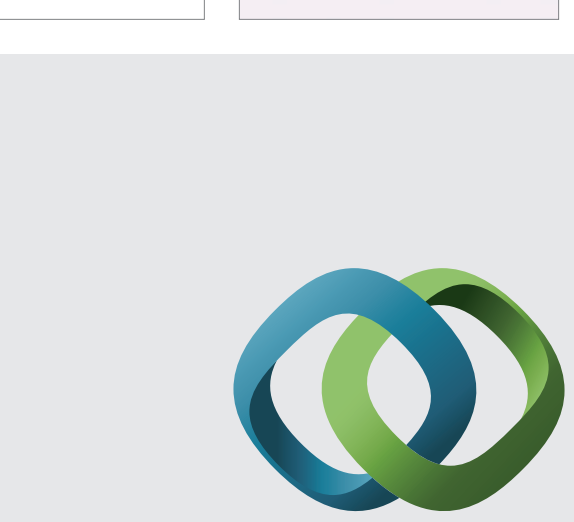

\section{Hindawi}

Submit your manuscripts at

http://www.hindawi.com
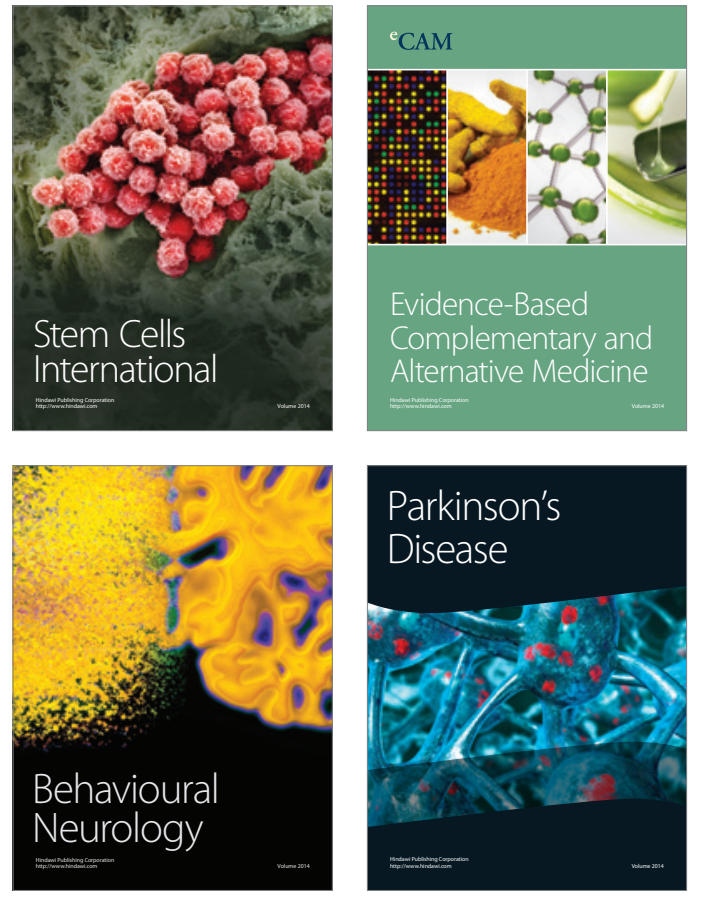
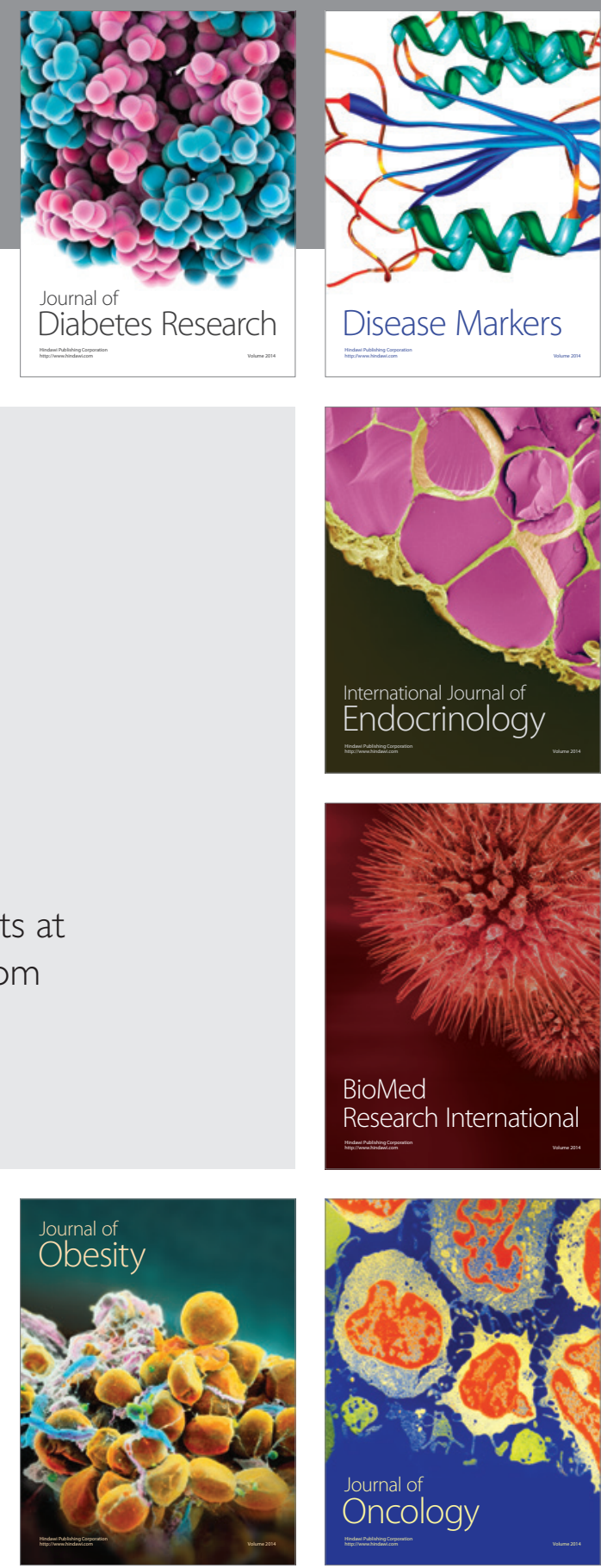

Disease Markers
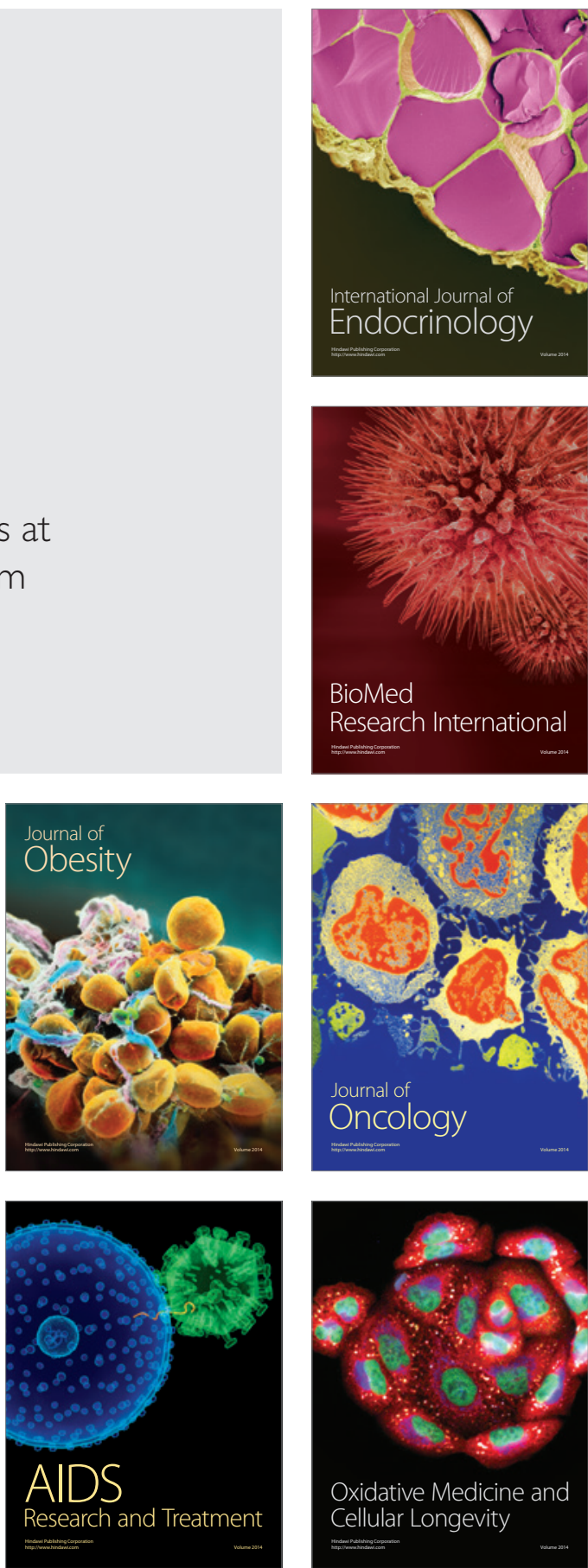